\title{
Introduction to the special edition from the Editors Pediatric Chiari I malformation-searching for clarity amidst myth, misnomer, and misunderstanding
}

\author{
Luca Massimi $^{1,2} \cdot$ Dominic N. P. Thompson $^{3}$ \\ Published online: 17 August 2019 \\ (C) Springer-Verlag GmbH Germany, part of Springer Nature 2019
}

The number of publications devoted to Chiari I malformation in childhood rises year on year. The number of subtypes of Chiari I malformation steadily expands. There has been a profusion of surgical options and treatment strategies available to the pediatric neurosurgeon. The increased availability of MRI and inconsistent diagnostic criteria have led to an abundance of children labeled "Chiari patients"; many of whom are asymptomatic or indeed do not have a Chiari at all!

The descriptive terms that we use to describe this phenomenon: Chiari malformation, Chiari anomaly, Chiari deformation, hindbrain hernia, tonsillar ectopia, low lying tonsils, to name but a few, serve to emphasize the etiological ambiguity surrounding this condition. Whatever description we choose to use it seems increasingly evident that the condition we are referring to bears little resemblance to that originally described by Chiari.

These are just some of the factors that have contributed to the trend toward confusion rather than clarity in respect of pediatric Chiari 1 malformation. It is regrettable that we still

Luca Massimi

1massmi@email.it

Dominic N. P. Thompson

dominic.thompson@gosh.nhs.uk

1 Neurochirurgia Infantile, Fondazione Policlinico Gemelli IRCCS, Rome, Italy

2 Istituto Neurochirurgia, Università Cattolica del Sacro Cuore, Rome, Italy

3 Department of Paediatric Neurosurgery, Great Ormond Street Hospital for Children NHS Trust, Great Ormond Street, London WC1N 3JH, UK lack an agreed definition for this entity. The arbitrary radiological definition " $5 \mathrm{~mm}$ tonsillar descent below the foramen magnum" lacks specificity or sensitivity and there is a pressing imperative to better define what we mean by Chiari I. Only then can we hope to better understand and address some of the controversies surrounding this enigmatic condition.

For all the above reasons, a special edition of Childs Nervous System devoted to Chiari I malformation is timely. An opportunity not of course to resolve these issues but to provide a forum in which we can highlight areas where there is understanding and consensus - as well as those where we do not, to share clinical experience and attitudes from around the world, and to expose areas fertile for future research.

The contributions to this Special Edition have been loosely grouped into three themes. Firstly, controversies, etiology, and patient evaluation. There is increasing recognition that Chiari $I$ is not a single diagnostic entity but rather the final common pathway for a variety of pathophysiological processes. Articles in this section critically appraise some of the pathophysiological processes deemed to underlie the Chiari I malformation including raised ICP and disorders of CSF circulation. The diverse clinical presentation of Chiari I malformation perhaps reflects the plethora of etiologies, what symptoms are pertinent, and can extra investigations such as sleep studies and intracranial pressure monitoring help refine patient selection? Secondly, imaging and surgical technique. What is the role of advanced imaging techniques and can these inform surgical decision making? The numerous variations on the surgical technique of foramen magnum decompression suggest that no single technique is perfect and that selection of technique needs to be able to optimize the difficult balance between surgical efficacy (in terms of symptom/ syrinx control) and minimal operative risk. Some poor outcomes from Chiari I surgery likely reflect a failure to 
appreciate underlying biomechanical abnormalities at the craniovertebral junction and the concept of complex Chiari is reviewed. The final section comprises institutional series and outcomes from around the globe, a contemporary, international snapshot of the state of the art in pediatric Chiari surgery.

We would like to thank all those who have contributed to this special edition. We sincerely hope that this collection of articles will provoke debate and begin to inform the development of future, ISPN-endorsed guidelines for the evaluation and management of Children with Chiari Malformation. Or at very least help to debunk some myths, correct some misnomers, and resolve some misunderstandings.

\section{Compliance with ethical standards}

Conflict of interest The authors do not have any financial interest to disclose.

Publisher's note Springer Nature remains neutral with regard to jurisdictional claims in published maps and institutional affiliations. 